\title{
Conversion of fatty aldehydes into alk (a/e)nes by in vitro reconstituted cyanobacterial aldehyde- deformylating oxygenase with the cognate electron transfer system
}

\author{
Jingjing Zhang ${ }^{1,2}$, Xuefeng $\mathrm{Lu}^{1^{*}}$ and Jian-Jun $\mathrm{Li}^{1^{*}}$
}

\begin{abstract}
Background: Biosynthesis of fatty alk(a/e)ne in cyanobacteria has been considered as a potential basis for the sunlight-driven and carbon-neutral bioprocess producing advanced solar biofuels. Aldehyde-deformylating oxygenase (ADO) is a key enzyme involved in that pathway. The heterologous or chemical reducing systems were generally used in in vitro ADO activity assay. The cognate electron transfer system from cyanobacteria to support ADO activity is still unknown.

Results: We identified the potential endogenous reducing system including ferredoxin ( $\mathrm{Fd})$ and ferredoxin-NADP ${ }^{+}$ reductase (FNR) to support ADO activity in Synechococcus elongatus PCC7942. ADO (Synpcc7942_1593), FNR (SynPcc7942_0978), and Fd (SynPcc7942_1499) from PCC7942 were cloned, overexpressed, purified, and characterized. ADO activity was successfully supported with the endogenous electron transfer system, which worked more effectively than the heterologous and chemical ones. The results of the hybrid Fd/FNR reducing systems demonstrated that ADO was selective against Fd. And it was observed that the cognate reducing system produced less $\mathrm{H}_{2} \mathrm{O}_{2}$ than the heterologous one by $33 \%$ during ADO-catalyzed reactions. Importantly, $k_{\text {cat }}$ value of ADO 1593 using the homologous Fd/FNR electron transfer system is 3.7-fold higher than the chemical one.

Conclusions: The cognate electron transfer system from cyanobacteria to support ADO activity was identified and characterized. For the first time, ADO was functionally in vitro reconstituted with the endogenous reducing system from cyanobacteria, which supported greater activity than the surrogate and chemical ones, and produced less $\mathrm{H}_{2} \mathrm{O}_{2}$ than the heterologous one. The identified Fd/FNR electron transfer system will be potentially useful for improving ADO activity and further enhancing the biosynthetic efficiency of hydrocarbon biofuels in cyanobacteria.
\end{abstract}

Keywords: Biofuels, Fatty alk(a/e)ne, Synechococcus elongatus PCC7942, Aldehyde-deformylating oxygenase, Ferredoxin, Ferredoxin-NADP ${ }^{+}$reductase, The cognate reducing system

\section{Background}

It is imperative to develop renewable biofuels due to concerns about climate change, the diminishing supplies of fossil fuels, and energy security [1-6]. With regard to the sustainability of biomass resources, cellulosic ethanol and microalgal biodiesel have been becoming more and more attractive [1,6,7]. Taking into account fuel

\footnotetext{
*Correspondence: Ivxf@qibebt.ac.cn; lijj@qibebt.ac.cn

'Key Laboratory of Biofuels, Shandong Provincial Key Laboratory of Energy Genetics, Qingdao Institute of Bioenergy and Bioprocess Technology, Chinese Academy of Sciences, No. 189 Songling Road, Qingdao 266101, China Full list of author information is available at the end of the article
}

performance, ideal fuels should have very similar energy content, storage and transportation properties, and combustion properties to current transportation fuels allowing them to be used in the existing gasoline, diesel, and jet engines [3]. Fatty-acid-derived biofuels fulfil these criteria, among which fatty-acid-derived alk(a/e)nes could be the ideal replacement for fossil-based fuel due to the fact that fatty alk $(\mathrm{a} / \mathrm{e})$ nes are the main components of conventional fuels such as gasoline, diesel, and jet fuel [4]. Therefore, it is of great importance to investigate biosynthesis of fatty alk(a/e)nes.

\section{Biomed Central}

(c) 2013 Zhang et al.; licensee BioMed Central Ltd. This is an Open Access article distributed under the terms of the Creative Commons Attribution License (http://creativecommons.org/licenses/by/2.0), which permits unrestricted use, distribution, and reproduction in any medium, provided the original work is properly cited. 
Fatty alk(a/e)nes are mainly produced by plants, insects, birds, green algae, and cyanobacteria [8]. Cyanobacteria are the advantageous organisms over others for industrial applications as they incorporate the favourable characteristics of prokaryotes and plants, which can efficiently convert solar energy and carbon dioxide into biofuels in one biological system [2,9-11]. Furthermore, the genetic engineering platform for cyanobacteria has been well established [2,9-11].

Fatty alk(a/e)nes produced by organisms are typified by an odd number of carbons. More and more evidence has indicated that a two-step pathway for fatty alk(a/e)ne biosynthesis exists, including: (1) reduction of fatty acylACP or -CoA into corresponding aldehyde by acyl-ACP reductase; (2) conversion of fatty aldehyde into alk(a/e) ne by aldehyde decarbonylase [12]. Since it has been observed that the C1-derived coproduct of the second step is carbon monoxide, the enzyme involved in that reaction has been tentatively designated as aldehyde decarbonylase [12]. Recently, Schirmer et al. identified two genes involved in alk(a/e)ne biosynthesis in cyanobacteria [8]. Since identification of the biosynthetic pathway of alk(a/e) ne in cyanobacteria, aldehyde decarbonylase has attracted particular interest in industry and academia for biofuel production due to the difficult and unusual reaction it catalyses. It has been proved that: (1) the C1-derived coproduct is formate instead of widely supposed carbon monoxide (Figure 1) [12]; (2) the aldehyde hydrogen is retained in formate and the hydrogen of the nascent methyl group originates at least in part from solvent $\left(\mathrm{H}_{2} \mathrm{O}\right)$ [12]; (3) oxygen is absolutely required for this apparently hydrolytic reaction, and one $\mathrm{O}$-atom is incorporated into formate, so it has been proposed that widely accepted aldehyde decarbonylase should be redesignated as aldehydedeformylating oxygenase (ADO) by Li et al. (Figure 1) $[13,14]$; (4) the auxiliary reducing system (biological or chemical) providing four electrons is absolutely needed for ADO activity (Figure 1) [8,14-16]; (5) based on the crystal structure of ADO from Prochlorococcus marinus MIT9313, ADO belongs to the ferritin-like non-heme dimetal-carboxylate enzyme family [8,17]; (6) Andre et al. reported that ADO is reversibly inhibited by $\mathrm{H}_{2} \mathrm{O}_{2}$ originating from poor coupling of reductant consumption with alkane formation, and the inhibition can be relieved

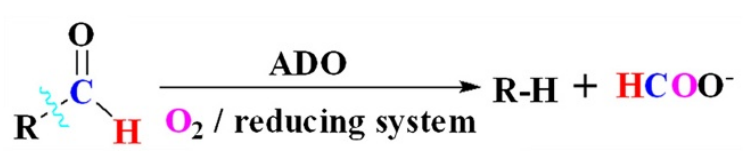

Figure 1 ADO-catalysed reaction [8,12-16]. Oxygen and the auxiliary reducing system (biological or chemical) providing four electrons are needed for ADO activity, one O-atom is incorporated into formate, and the aldehyde hydrogen is retained in formate. by supplementing catalase (The paper was published when the manuscript was under review.) [18].

As mentioned above, the biological or chemical reducing system is required for catalytic activity of $\mathrm{ADO}[8,14-16]$. The commonly used chemical reducing system is phenazine methosulfate (PMS) or 1-methyoxy-5-methylphenazinium methylsulfate (MeOPMS) and NADH [14-16]. The widely used biological reducing system is surrogate ferredoxin (Fd) and ferredoxin-NADP ${ }^{+}$reductase (FNR) from spinach, and NADPH $[8,14-16]$. It was observed that the chemical reducing system worked better than the biological one [14,15]. Fd from Zea mays and FNR from Anabaena sp. PCC7120 have also been used to support ADO from Prochlorococcus marinus MIT9313 [18].

In addition to supporting ADO ( Fd and FNR from spinach, Fd from Zea mays and FNR from Anabaena sp. PCC7120), the surrogate electron transfer systems also supported reactions catalysed by stearoyl acyl carrier protein $\Delta^{9}$ desaturase (Fd and FNR from spinach) [19], $p$-aminobenzoate $N$-oxygenase (AurF) (Fd and FNR from Anabaena sp. PCC7119) [20], and cytochrome P450 (P450) $[21,22]$, etc.. P450 showed enormous diversity in the redox partner systems [23]. The homologous Fd/FNR systems generally supported greater P450 activity than the heterologous ones, implying that the interaction of Fd with FNR and P450 is very important for efficient electron transfer [24-26].

Considering that the surrogate electron transfer system might not be well matched with cyanobacterial ADO, it is very necessary to search for the cognate one to support ADO. In this paper, ADO and the endogenous reducing system including Fd and FNR from Synechococcus elongatus PCC7942 were cloned, overexpressed, purified, and characterized. We reported the first cognate reducing system from cyanobacteria to support ADO activity (Figure 2). ADO was successfully in vitro reconstituted with the endogenous Fd/FNR system, which performed more effectively than the surrogate Fd/FNR one and the chemical reducing system.

\section{Results and discussion}

Searching for the endogenous electron transfer system to support ADO activity in the genome sequence of

\section{Synechococcus elongatus PCC7942}

According to the report by Schirmer et al., in vitro enzymatic activity of ADO was only observed in the presence of Fd, FNR, and NADPH, while omitting any one of these cofactors completely abolished ADO activity [8]. However, the endogenous electron transfer system from cyanobacteria to support ADO-catalysed reaction is still unknown. In order to in vitro reconstitute ADO activity, it is essential to search for such a cognate electron transfer system to be well matched with ADO in cyanobacteria.

Two FNR isoforms were isolated in Synechocystis sp. PCC6803, the smaller one $\left(F_{N}\right)$ similar to the one 


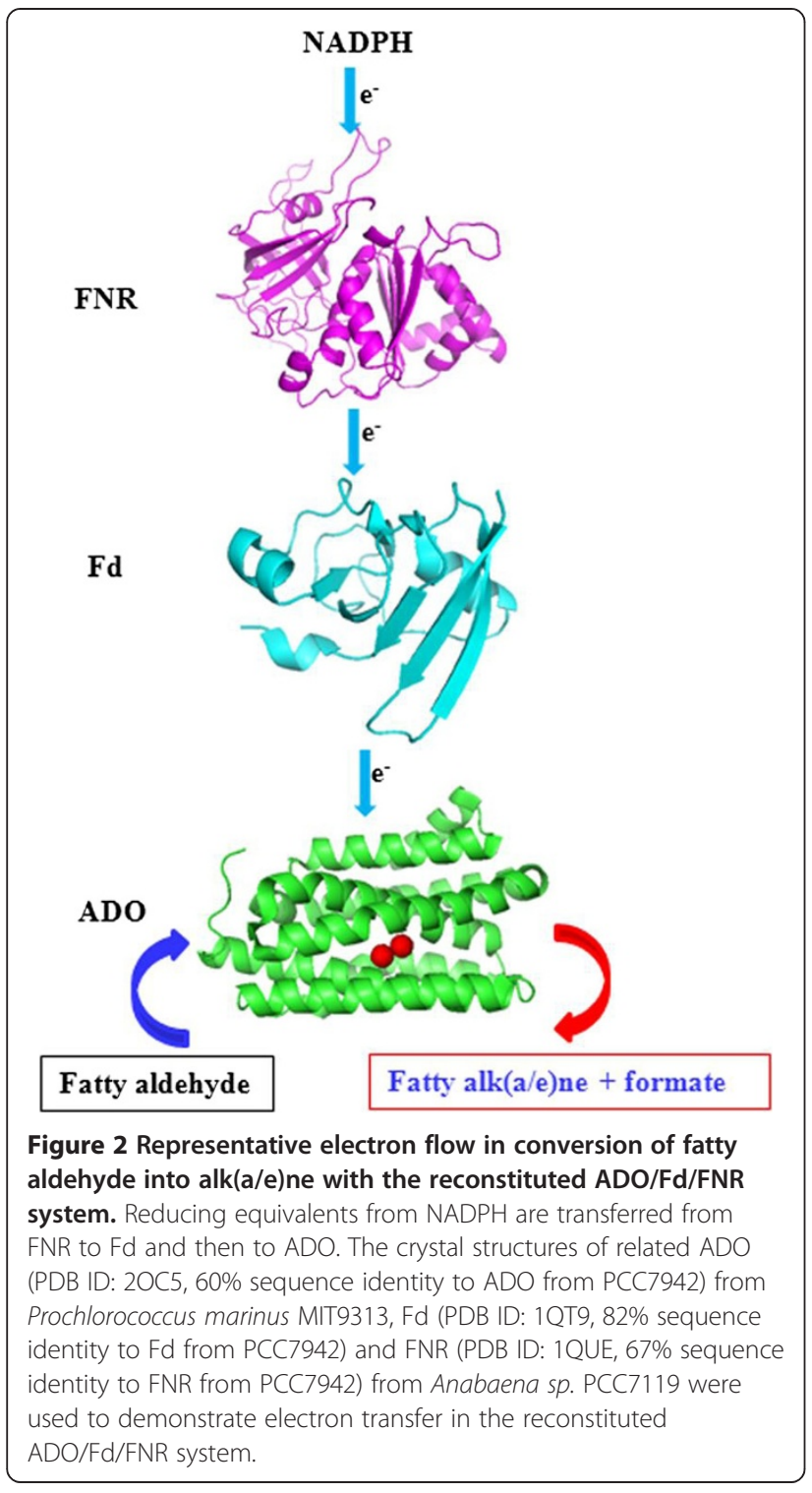

found in plant plastids and the larger one $\left(\mathrm{FNR}_{\mathrm{L}}\right)$ associated with the phycobilisome, both of which derive from the single FNR gene (petH), and the smaller one is produced from the second translation initiation site within the petH ORF [27,28]. Two FNR isoforms were also detected in Anabaena sp. PCC7120 and Synechococcus $s p$. PCC7002 [28]. However, the smaller isoform is not present in cyanobacteria lacking a second methionine within the petH ORF such as Synechococcus elongatus and Thermosynechococcus elongatus [28], so only one FNR isoform is present in PCC7942. According to the genome sequence of PCC7942 (http://genome.microbedb. jp/cyanobase/SYNPCC7942), FNR is encoded by the SynPcc7942_0978 (petH) gene, and would be involved in electron transfer between FNR and its protein partners in PCC7942.
There are at least seven Fd and Fd-like proteins in the genome sequence of PCC7942 (http://genome. microbedb.jp/cyanobase/SYNPCC7942), among which Ferredoxin I (SynPcc7942_1499) encoded by the petF gene was found to be indispensable for PCC7942 [29]. In addition, in PCC6803 the corresponding petF gene (ssl0020) coding for the most abundant ferredoxin product was also proved to be critical to cell growth, and the expression level of the petF-like genes such as sll1382, slr0150 and slr1828 is very weak compared with that of petF [30]. So, we think that Fd encoded by the petF gene is very important for a lot of redox processes in cyanobacteria, such as mediating electron transfer from ironsulphur centres of photosystem I to FNR which then reduces $\mathrm{NADP}^{+}$for $\mathrm{CO}_{2}$ fixation, cyclic photophosphorylation, nitrogen assimilation, sulphite reduction or fatty acid metabolism, etc. Based on these considerations, $\mathrm{Fd}$ (SynPcc7942_1499) encoded by the petF gene was chosen for mediating electron transfer between FNR and ADO.

Therefore, FNR (SynPcc7942_0978) and Ferredoxin I (SynPcc7942_1499) will be investigated to support ADO from $\mathrm{PCC} 7942$ in the paper.

\section{Cloning, overexpression, purification, and characterization} of FNR from Synechococcus elongatus PCC7942

The gene encoding FNR (SynPcc7942_0978) was amplified from genomic DNA of PCC7942 by PCR, cloned into the vector pET-28a $(+)$ at the restriction sites of NdeI and XhoI, and overexpressed in E. coli BL21(DE3) under IPTG induction. Overexpressed FNR with the $\mathrm{N}$-terminal His-tag was purified to homogeneity on Nickel column (Additional file 1: Figure S1). The predicted molecular weight of FNR is $44.4 \mathrm{kDa}$, corresponding very well to SDS-PAGE (Additional file 1: Figure S1). Protein yield was about $12 \mathrm{mg} / \mathrm{L}$.

FNRs, usually obtained in the oxidized state, contain the noncovalently bound FAD cofactor. The released FAD from the $\mathrm{FNR}_{\mathrm{L}}$-phycocyanin complex (the larger FNR isoform, 63\% sequence identity to FNR from PCC7942) of PCC6803 was recovered and quantified [27]. What's more, based on the crystal structure of FNR (PDB ID:2B5O, 61\% sequence identity to FNR from PCC7942) from Synechocystis $s p$. PCC7002 with FAD bound, the residues involved in FAD binding were identified, including Arg179, Leu180, Tyr181, Ser182, Cys200, Arg202, Leu204, Tyr206, Gln207, Val218, Cys219, Ser220, Thr260, Tyr402, which are completely conserved in FNRs from PCC 7942 and PCC6803 (Additional file 2). Therefore FNR from PCC7942 should have the characteristic FAD absorption spectrum. The UV/vis spectrum of FNR clearly showed two peaks at 385 and $455 \mathrm{~nm}$, and a shoulder at $480 \mathrm{~nm}$, demonstrating that FNR is certainly a flavoprotein (Figure 3A) [31,32].

FNR was assayed through ferricyanide reductase activity. This assay (also called diaphorase activity) was used to 


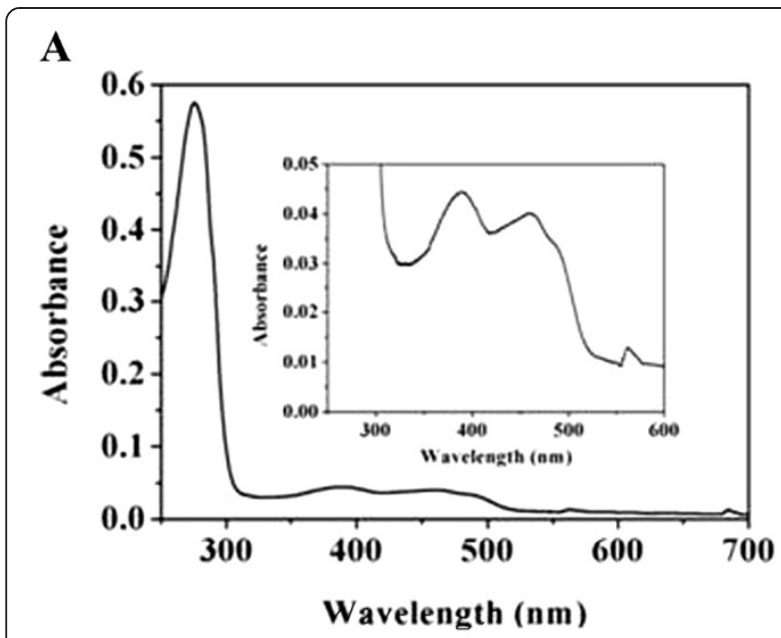

B

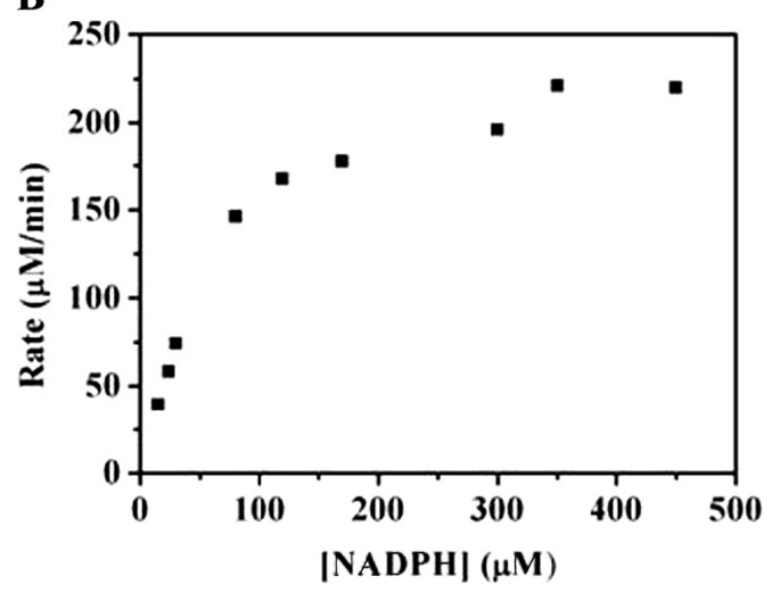

Figure 3 Characterization of FNR from Synechococcus elongatus PCC7942. (A) UV-vis absorption spectrum of FNR. (B) Ferricyanide reductase activity of FNR.

determine kinetic parameters of FNR in the presence of the electron acceptor potassium ferricyanide. FNR from PCC7942 was active against potassium ferricyanide, and the initial rates were plotted against NADPH concentration and fitted according to the Michaelis-Menten equation (Figure 3B). Kinetic parameters of FNRs from different cyanobacteria were listed in Table 1. Compared with two FNR isoforms from PCC6803, FNR from PCC7942 showed higher $K_{m(N A D P H)}$ and $k_{c a t}$ values, but slightly lower catalytic efficiency [27]. In comparison with FNR from Anabaena sp. PCC7119, FNR from PCC7942 exhibited higher $K_{m(N A D P H)}$ and similar $k_{c a t}$ values, but much lower catalytic efficiency [33]. These results indicated that FNRs from the evolutionarily diverse classes of cyanobacteria had different binding affinity for NADPH and catalytic efficiency towards the ferricyanide reduction. In addition, it was observed that the specific activity of FNR using NADPH $(375 \mu \mathrm{M})$ was 23 -fold higher than NADH (2 mM), demonstrating that, like other FNRs [31,34], FNR from PCC7942 also prefers NADPH over NADH.

Cloning, overexpression, purification, and characterization of Fd from Synechococcus elongatus PCC7942

The petF gene encoding Fd (SynPcc7942_1499) was amplified from genomic DNA of PCC7942 by PCR, cloned into the vector pET-28a $(+)$ at the restriction sites of $\mathrm{NdeI}$ and XhoI, and overexpressed in E. coli BL21(DE3) in M9 medium under IPTG induction at $30^{\circ} \mathrm{C}$ [35]. Overexpressed Fd was purified on Nickel column. The predicted molecular weight of Fd with the N-terminal His-tag is $12.8 \mathrm{kDa}$, which is much smaller than the estimated one (above $20 \mathrm{kDa}$ ) by SDS-PAGE (Additional file 1: Figure S2). This is probably due to the acidic character of Fd (predicted pI 3.8), which prevents proper binding of SDS [36]. Fd expression was also confirmed by Western blot (data not shown). Protein yield was about $5 \mathrm{mg} / \mathrm{L}$.

Based on analytical ultracentrifugation analysis, the estimated molecular weight of $\mathrm{Fd}$ is about $11.8 \mathrm{kDa}$ after the $\mathrm{N}$-terminal His-tag was removed with thrombin (Four amino acids GSHM were left at the N-terminal.), demonstrating that Fd is a monomer. MALDI-TOF-MS showed the molecular weight is $10932.79 \mathrm{Da}$ after tag removal, corresponding to the predicted molecular weight 10933.02 Da. In addition, the UV/vis spectrum of Fd clearly showed three peaks characteristic of the presence of the [2Fe-2S] cluster at 331, 423, and $463 \mathrm{~nm}$ (Figure 4A) [36,37].

The function of Fd was investigated through Fd-mediated cytochrome $c$ reductase activity of FNR. This assay was used to determine the affinity for Fd and $k_{c a t}$ value of FNR in the presence of its cognate electron acceptor $\mathrm{Fd}$, and to see if the cognate FNR/Fd system from PCC7942 can be functionally coupled for efficient electron transfer.To get more information about the Fd reduction, the initial rates were obtained by varying Fd concentration under saturating concentrations of NADPH and cytochrome $c$. The initial rates of the cytochrome $c$ reduction were plotted against Fd concentration and fitted according to the Michaelis-Menten equation (Figure 4B). $K_{m(F d)}, k_{c a t}$ and $k_{\text {cat }} / K_{m}$ values of FNR against the Fd-mediated cytochrome $c$ reduction were $15.9 \mu \mathrm{M}, 85.6 \mathrm{~s}^{-1}$, and $5.4 \mu \mathrm{M}^{-1} \mathrm{~s}^{-1}$ respectively, indicating that the cognate FNR/Fd electron transfer system from PCC7942 can be efficiently and functionally coupled for the cytochrome $c$ reduction. In contrast with two FNR isoforms from PCC6803, FNR from PCC7942 showed higher affinity for $\mathrm{Fd}$, lower $k_{\text {cat }}$ value, and similar catalytic efficiency to $\mathrm{FNR}_{\mathrm{s}}$, but higher catalytic efficiency than $\mathrm{FNR}_{\mathrm{L}}-\mathrm{PC}$ (Table 1) [27]. Compared with FNR from PCC7119, FNR from PCC7942 exhibited similar affinity for Fd, much lower $k_{\text {cat }}$ value and catalytic efficiency (Table 1) [33]. These results also demonstrated that FNRs from different cyanobacteria exhibited different kinetic behaviour against the Fd-mediated reduction of cytochrome $c$. 
Table 1 Comparison of kinetic parameters of FNRs from different cyanobacteria

\begin{tabular}{|c|c|c|c|c|c|c|c|}
\hline \multirow[b]{2}{*}{ FNR } & \multicolumn{3}{|c|}{ Diaphorase activity } & \multicolumn{3}{|c|}{ Cytochrome $c$ reductase activity } & \multirow[b]{2}{*}{ Reference } \\
\hline & $\begin{array}{l}K_{m(N A D P H)} \\
(\mu \mathrm{M})\end{array}$ & $\begin{array}{l}k_{\text {cat }} \\
\left(\mathrm{s}^{-1}\right)\end{array}$ & $\begin{array}{l}k_{c a t} / K_{m} \\
\left(\mu \mathrm{M}^{-1} \mathrm{~s}^{-1}\right)\end{array}$ & $\begin{array}{l}K_{m(F d)} \\
(\mu \mathrm{M})\end{array}$ & $\begin{array}{l}k_{\text {cat }} \\
\left(s^{-1}\right)\end{array}$ & $\begin{array}{l}k_{\text {cat }} / K_{m} \\
\left(\mu \mathrm{M}^{-1} s^{-1}\right)\end{array}$ & \\
\hline PCC7942 & $92.5 \pm 7.6$ & $227 \pm 7$ & $2.5 \pm 0.3$ & $15.9 \pm 1.2$ & $85.6 \pm 3$ & $5.4 \pm 0.5$ & this study \\
\hline FNR $-P C$ from PCC6803 & $40 \pm 3$ & $124 \pm 3$ & $3.1 \pm 0.3$ & $47 \pm 6$ & $144 \pm 12$ & $3.1 \pm 0.7$ & {$[27]$} \\
\hline $\mathrm{FNR}_{5}$ from PCC6803 & $55 \pm 5$ & $174 \pm 5$ & $3.2 \pm 0.4$ & $28 \pm 2$ & $154 \pm 6$ & $5.5 \pm 0.6$ & {$[27]$} \\
\hline PCC7119 & $23.0 \pm 1.2$ & $225 \pm 3$ & $9.8 \pm 0.2$ & $11.0 \pm 2.0$ & $200 \pm 10$ & $18.2 \pm 1.0$ & [33] \\
\hline
\end{tabular}

Cloning, overexpression, purification, and characterization of ADO from Synechococcus elongatus PCC7942

The codon-optimized gene encoding ADO (Synpcc7942_ 1593) from PCC7942 was cloned into the vector pET$28 \mathrm{a}(+)$ at the restriction sites of NdeI and XhoI, and successfully overexpressed in E. coli BL21(DE3) under IPTG

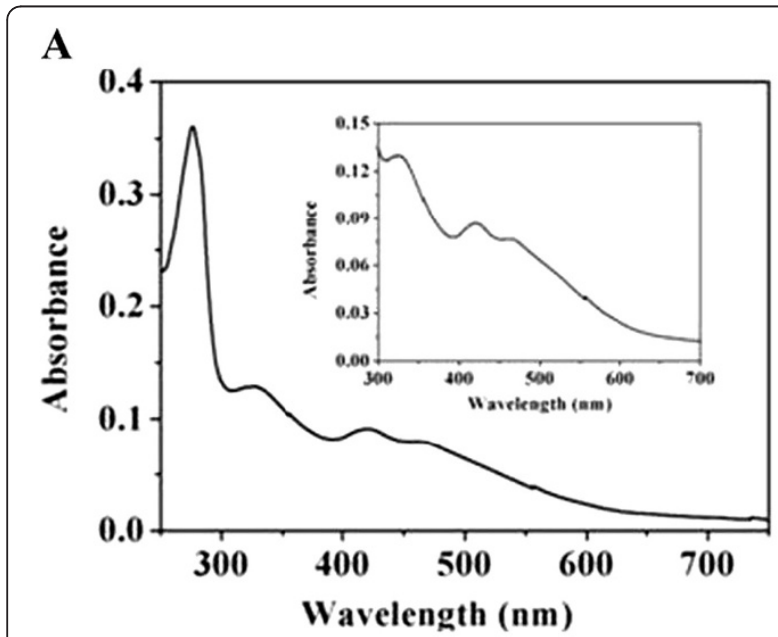

B

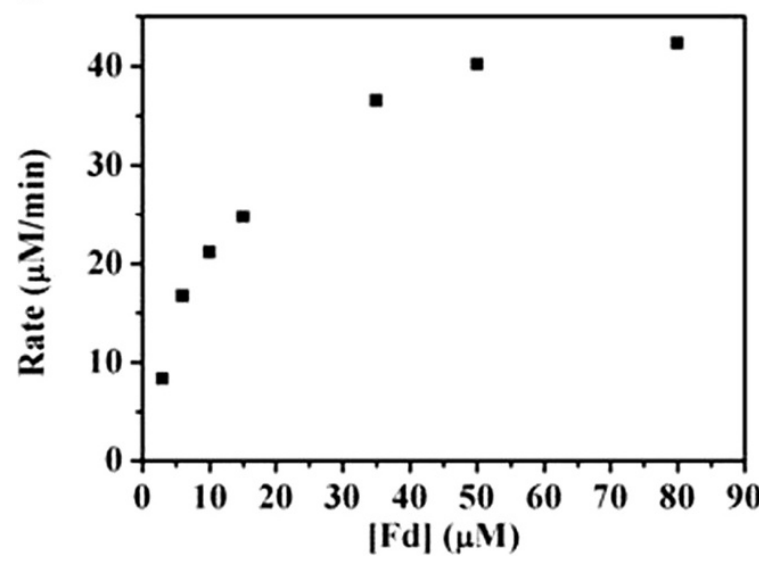

Figure 4 Characterization of Fd from Synechococcus elongatus PCC7942. (A) UV-vis absorption spectrum of Fd. (B) Fd-mediated cytochrome $c$ reductase activity of FNR. induction. Overexpressed ADO 1593 was purified to homogeneity by Nickel-chelating affinity chromatography. The target protein was eluted with buffers containing 60 and $80 \mathrm{mM}$ imidazole (Figure 5). The predicted molecular weight of ADO 1593 is $26.4 \mathrm{kDa}$, corresponding well to SDS-PAGE (Figure 5). The collected fractions containing the target protein were combined and treated as reported $[15,16]$. Protein yield was about $15 \mathrm{mg} / \mathrm{L}$.

Long and short-chain aldehydes such as n-octadecanal and $n$-heptanal were generally used as the substrates for ADO assay [8,12-16]. However, we found that $n$-hexadecanal is a better substrate than $n$-octadecanal in terms of solubility and activity (unpublished result). According to the recent paper by Andre et al., actually ADO doesn't show any strong chain-length specificity against its substrates (C8 to C18) [18]. So both $n$-hexadecanal and $n$-heptanal were tested as the substrates for ADO 1593 in the paper. Just like other ADOs, ADO 1593 was also active against these two substrates in the presence of the chemical reducing system (PMS and NADH) (data not shown).

\section{Comparing the effects of different reducing systems on ADO activity}

Using n-hexadecanal as the substrate

We were inspired by the Fd-mediated the cytochrome $c$ reduction with FNR (Figure 4B), and ADO 1593 was

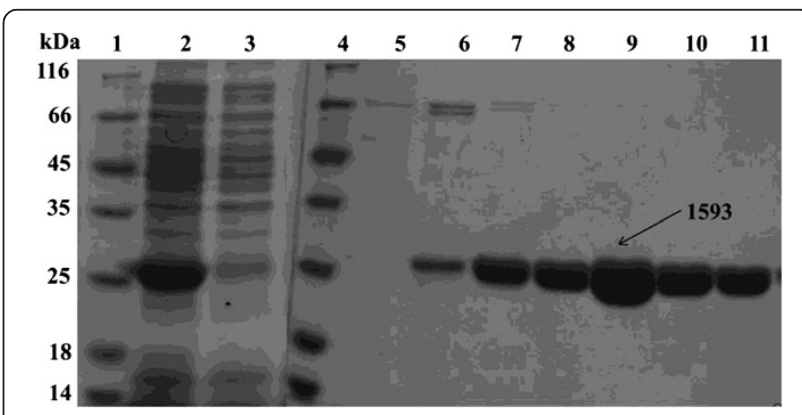

Figure 5 SDS-PAGE analysis of ADO 1593. Lane 1 and 4, low protein molecular weight marker; Lane 2, crude supernatant for over-expressed AD 1593; Lane 3, flow-through when loaded; Lane 5 and 6, eluents of buffer A $+60 \mathrm{mM}$ imidazole; Lanes 7-11, eluents of buffer $\mathrm{A}+80 \mathrm{mM}$ imidazole. 
in vitro reconstituted with Fd and FNR from PCC7942. Interestingly, $n$-hexadecanal was successfully converted into $n$-pentadecane by in vitro reconstituted ADO 1593 with the cognate Fd/FNR system. The performance of the cognate electron transfer system in conversion of $n$-hexadecanal was then compared with the surrogate Fd/FNR system from spinach and the chemical one (PMS/NADH). The hybrid systems consisting of $\mathrm{Fd}_{\mathrm{PCC} 7942} /$ $\mathrm{FNR}_{\text {spinach }}$ and $\mathrm{Fd}_{\text {spinach }} / \mathrm{FNR}_{\mathrm{PCC} 7942}$ were also investigated (Table 2). Assays were carried out with FNR/Fd/ADO 1593 ratios of 1:5.7:11.1 as a compromise for minimizing the yield variations with changes in the ratios [24,25].

The experimental results were shown in Table 2, and at least three conclusions were drawn as listed here: (1) The biological reducing system including the cognate and surrogate ones is superior to the chemical one, which is contradictory to the published results, especially for the heterologous Fd/FNR system from spinach. The different conclusion might arise from the fact that more spinach FNR up to $1.9 \mathrm{U} / \mathrm{ml}$ was used in our assay, whereas much less FNR $(0.1 \mathrm{U} / \mathrm{ml}$ or $0.04 \mathrm{U} / \mathrm{ml}$, equivalent to less than ours by 19 or 47.5 -fold) was used in the literature $[14,15]$. Whereas Andre et al. found that no more products formed when more FNR or Fd was added to an exhausted in vitro ADO reaction, more products were produced only when more ADO was added [18]. Considering that ADO had been completely inactivated by in situ-produced $\mathrm{H}_{2} \mathrm{O}_{2}$, this is understandable. However, the scenario is different in our case, since more FNR and Fd were used when ADO-catalyzed reactions were initiated. (2) The cognate biological reducing system is more effective than the surrogate one. $n$-Pentadecane yield using the cognate Fd/FNR system from PCC7942 is $26.6 \%$ higher than the surrogate one from spinach, implying that the interaction of Fd with FNR and ADO is very important in supporting $\mathrm{ADO}$ activity and mediating efficient electron transfer between Fd and its redox partners FNR and ADO.

Table 2 Comparison of the effects of different reducing systems on ADO activity

\begin{tabular}{lll}
\hline \multicolumn{2}{l}{ Reducing system } & $\begin{array}{l}\text { Yield of } \boldsymbol{n} \text {-pentadecane } \\
(\boldsymbol{\mu} \mathrm{M})\end{array}$ \\
\hline Chemical & $\mathrm{PMS}+\mathrm{NADH}$ & $8.6 \pm 0.1$ \\
\hline \multirow{3}{*}{ Biological } & $\mathrm{Fd}_{\text {spinach }}+\mathrm{FNR}_{\text {spinach }}+\mathrm{NADPH}$ & $15.7 \pm 0.5$ \\
& $\mathrm{Fd}_{\text {spinach }}+\mathrm{FNR}_{\mathrm{PCC7942}}+\mathrm{NADPH}$ & $13.9 \pm 0.4$ \\
& $\mathrm{Fd}_{\mathrm{PCC7942}}+\mathrm{FNR}_{\text {spinach }}+\mathrm{NADPH}$ & $17.6 \pm 0.3$ \\
& $\mathrm{Fd}_{\mathrm{PCC7942}}+\mathrm{FNR}_{\mathrm{PCC7942}}+\mathrm{NADPH}$ & $20.0 \pm 0.8$ \\
\hline
\end{tabular}

The reaction mixtures contained $20 \mu \mathrm{M}$ ADO 1593, $80 \mu \mathrm{M}$ ferrous ammonium sulfate, $150 \mu \mathrm{M} n$-hexadecanal, $75 \mu \mathrm{M}$ PMS and $750 \mu \mathrm{M} \mathrm{NADH}$, or $10.26 \mu \mathrm{M}$ Fd from spinach or PCC7942 and $1.8 \mu \mathrm{M}$ FNR from spinach or PCC7942 and $2 \mathrm{mM}$ $\mathrm{NADPH}$, in $500 \mu \mathrm{L}$ of $100 \mathrm{mM}$ HEPES (pH 7.2) containing $0.1 \mathrm{M} \mathrm{KCl}$ and $10 \%$ glycerol. The reactions were incubated at room temperature for $1 \mathrm{hr}$ at $200 \mathrm{rpm}$, and the products were extracted with $500 \mu \mathrm{L}$ ethyl acetate for GC-MS analysis.
Similar results have been reported for some P450s. For example, the cognate Fd/FNR system from Sphingomonas sp. strain AO1 is more effective in supporting P450 than the heterologous one from spinach [26]; Ewen et al. recently reported that the protein-protein recognition in the mitochondrial cytochrome P450 system and modulation of electron transfer between Adx (adrenodoxin) and its redox partners AdR (adrenodoxin reductase) and cytochrome P450 are essential for mammalian cytochrome $\mathrm{P} 450$ [38]; When the P450 enzymes from $N$. aromaticivorans were reconstituted with the cognate ArR (Fd reductase) and Arx (a [2Fe-2S] Fd), the steady-state turnover rates increased by $50 \%$ to $400 \%$ over those observed previously with the surrogate PdR (putidaredoxin reductase) [24,25]. (3) The hybrid experiments of the surrogate and the cognate biological reducing systems demonstrated that ADO is selective against Fd and the interaction between Fd and FNR is very important for efficient electron transfer and ADO activity. It has been observed that the interactions between FNR and its protein partner Fd or flavodoxin are essential for efficient assembling and functionality of the formation of FNR-Fd complex [39]. A lot of examples have demonstrated that the interactions between P450s and Fd proteins are significant for P450 activity. For instance, Bell et al. tailored an nonphysiological Fd to support native-like P450 activity through engineering the surface residues involved in the interaction between Fd and P450 [40]; P450 105D5 from Streptomyces coelicolor A3(2) is very selective among S. coelicolor Fd proteins, but Fd could interact with the surrogate FNRs from P. putida and spinach [22]; With S. griseolus P450 105A1 and 105B1, either of two Fd proteins could reconstitute P450 activity, but each Fd worked at least somewhat faster with its cognate P450 [41].

\section{Using n-heptanal as the substrate}

$k_{\text {cat }}$ values of ADO 1593 against $n$-heptanal were determined using the cognate reducing system and the chemical one respectively under saturating concentration of $n$-heptanal (2 mM, Estimated $K_{m}$ value of ADO1593 for $n$-heptanal was $224 \pm 40 \mu \mathrm{M}$ employing the chemical reductants.). ADO 1593 had $k_{\text {cat }}$ value of $0.44 \pm 0.02 \mathrm{~min}^{-1}$ in the presence of the homologus Fd/FNR system, whereas $k_{\text {cat }}$ value was $0.12 \pm 0.02 \mathrm{~min}^{-1}$ for the NADH/PMS system, close to that of ADO from Prochlorococcus marinus $\left(0.17 \pm 0.01 \mathrm{~min}^{-1}\right)$ [16]. Thus, the turnover number of ADO 1593 using the cognate Fd/FNR electron transfer system is 3.7-fold higher than the chemical one. However, according to the report by Choi et al., AurF ( $p$ aminobenzoate $N$-oxygenase) showed similar $k_{\text {cat }}$ values when reconstituted with either the chemical reductants (NADH/PMS) or the biological one (Fd/FNR from Anabaena sp. PCC7119) [20]. Their results could arise 
from the fact that the surrogate Fd/FNR reducing system was used, again highlighting the importance of the homologous electron transfer system in supporting greater enzymatic activity.

\section{$\mathrm{H}_{2} \mathrm{O}_{2}$ production during in vitro $A D O$-catalyzed reactions}

Andre et al. suggest that the inhibitory $\mathrm{H}_{2} \mathrm{O}_{2}$ formation is due to uncoupled electron transfer from NADPH to $\mathrm{O}_{2}$ [18]. We directly compared $\mathrm{H}_{2} \mathrm{O}_{2}$ concentration for the cognate electron transfer system and the heterologous one using $n$-hexadecanal as the substrate in order to see whether there is any difference in $\mathrm{H}_{2} \mathrm{O}_{2}$ production between these two biological reducing systems. Interestingly, we observed that the cognate reducing system produced less $\mathrm{H}_{2} \mathrm{O}_{2}$ than the heterologous one by $33 \%$, demonstrating that better coupling between FNR:Fd:ADO in the cognate reducing system might lead to more efficient electron transfer and therefore less formation of $\mathrm{H}_{2} \mathrm{O}_{2}$ due to decoupled electron transfer to $\mathrm{O}_{2}$. This may be one reason why the cognate electron transfer system used yields more product than the heterologous one.

\section{Conclusions}

The cognate electron transfer system including Fd and FNR from cyanobacteria to support ADO activity was identified, cloned, overexpressed, purified, and characterized. For the first time, ADO was functionally in vitro reconstituted with the endogenous reducing system from cyanobacteria, which supported higher ADO activity than the surrogate Fd/FNR system and the chemical one, and produced less $\mathrm{H}_{2} \mathrm{O}_{2}$ than the heterologous one. The identified Fd/FNR reducing system offers the platform to study the Fd-ADO recognition and electron transfer in detail, will be potentially useful for further improving ADO activity, and might be applicable to other enzymes requiring the electron transfer system. Our findings here might be significant for further building a more active in vivo fatty alk(a/e)ne-biosynthesis system in cyanobacteria and constructing a highly efficient photosynthetic microbial cell factory for production of advanced hydrocarbon biofuels.

\section{Methods}

\section{Materials}

Spinach ferredoxin and ferredoxin-NADP ${ }^{+}$reductase, horse-heart cytochrome $c$, BSA (Bovine Serum Albumin), NADPH, NADH, $n$-heptanal, potassium ferricyanide, phenazine methosulfate (PMS), ferrous ammonium sulfate, Dess-Martin reagent, $n$-hexadecanol were obtained from Sigma-Aldrich. Oligonucleotides were synthesized by Shanghai Sangon Biotech Co. Ltd (China). The gene encoding ADO (Synpcc7942_1593) from Synechococcus elongatus PCC7942 with codon optimization was synthesized and cloned into the vector pBluescript II SK(+) using the restriction sites of XhoI (3'-terminal) and
SmaI (5'-terminal) by Shanghai Sangon Biotech Co. Ltd (China) (Additional file 3), and the NdeI restriction site was introduced at 5'-terminal. Taq DNA polymerases and all restriction endonucleases were from Fermentas or Takara Biotechnology. The kits used for molecular cloning were from Omega Bio-tek or Takara Biotechnology. Nickel column and the expression vectors were from Novagen. Antibodies and chemical reagents used for Western blot were from Tiangen (China). Amicon YM10 membrane was from Millipore. The Amplex Red Hydrogen Peroxide/Peroxidase Assay Kit was purchased from Invitrogen.

\section{Bacterial strains, plasmids, and media}

E. coli DH5 $\alpha$ was used for routine DNA transformation and plasmid isolation. E. coli BL21(DE3) was utilized for protein overexpression. E. coli strains were routinely grown in Luria-Bertani broth at $37^{\circ} \mathrm{C}$ with aeration or on $\mathrm{LB}$ supplemented with $1.5 \%$ (w/v) agar. $50 \mu \mathrm{g} / \mathrm{ml}$ Kanamycin was added when required. The vector pET-28a(+) was used for subcloning.

\section{DNA manipulations}

General molecular biology techniques were carried out following the standard procedures [42]. Restriction and modification enzymes were used following the recommendations of the manufacturers. DNA fragments were purified from agarose gels using the DNA gel extraction kit. Plasmid DNA was isolated using the plasmid miniprep kit.

The plasmid, where the codon-optimized gene encoding ADO (Synpcc7942_1593) from PCC7942 was inserted into the vector pBluescript II SK(+), was digested with $N d e I$ and $X h o I$, and re-cloned into the vectorspET-28a(+) digested with same restriction enzymes.

The gene encoding Fd SynPcc7942_1499 was amplified with the forward primer (5'-GCTCAGCATATGATGGCA ACCTACAAGG-3', the NdeI I restriction site underlined) and the reverse primer (5'-GGCTCGCTCGAGTTAGTAG AGGTCTTCTTC-3', the XhoI restriction site underlined) using genomic DNA as a template. The gene encoding FNR SynPcc7942_0978 was amplified with the forward primer (5' -CGCGGCCATATGATGTTGAATGCGAGT GTG-3', the NdeI I restriction site underlined) and the reverse primer $\left(5^{\prime}\right.$-CATTCGCTCGAGGGCTGAACTA GTAGGTTT-3', the XhoI restriction site underlined) using genomic DNA as a template. The PCR products were isolated by agarose electrophoresis and extracted from agarose gel using the DNA gel extraction kit, digested with restriction enzymes $\mathrm{NdeI}$ and $\mathrm{XhoI}$ respectively, and re-cloned into the vector pET-28a(+) digested with $N d e \mathrm{I}$ and XhoI, respectively. All constructs were confirmed by DNA sequencing. 


\section{Protein overexpression and purification}

The expression constructs pET28a-1593 and pET28aFNR were transformed into competent $E$. coli BL21(DE3). Protein expression was carried out at $37^{\circ} \mathrm{C}$ in $\mathrm{LB}$ media supplemented with $50 \mu \mathrm{g} / \mathrm{mL}$ kanamycin. The cultures were induced with $1 \mathrm{mM}$ IPTG when $\mathrm{OD}_{600}$ reached 0.7 , and were shaken at $37^{\circ} \mathrm{C}$ for additional 3 hours. The histagged proteins were purified using Nickel chelating resin according to the manufacturer's protocol. Apo-ADO was prepared according to the published procedure, and the diferrous form of ADO was reconstituted by the addition of the stoichiometric amounts of ferrous ammonium sulfate to apo-ADO prior to assay $[15,16]$. Proteins were concentrated with Amicon YM10 membrane (10 kDa cut-off). Protein concentration was determined by the Bradford method using bovine serum albumin as a standard.

The expression construct pET28a-Fd was expressed in E. coli BL21(DE3). Protein expression was carried out at $30^{\circ} \mathrm{C}$ in $\mathrm{M} 9 \mathrm{ZB}$ media supplemented with $50 \mu \mathrm{g} / \mathrm{mL}$ kanamycin and $50 \mu \mathrm{M} \mathrm{FeCl}{ }_{3}$ [35]. The cultures were induced with $0.2 \mathrm{mM}$ IPTG when $\mathrm{OD}_{600}$ reached 0.7 , and were shaken at $16^{\circ} \mathrm{C}$ for $48 \mathrm{hr}$. His-tagged Fd was purified as above.

SDS-PAGE was performed in $12 \%$ polyacrylamide gels, using the low protein molecular weight marker and Coomassie Blue R-250 staining. For Western blot, proteins were transferred from the gel onto the polyvinylidene fluoride (PVDF) membrane using the Mini Trans-Blot Electrophoretic Transfer Cell. The membrane was blocked with $5 \%(\mathrm{w} / \mathrm{v})$ skimmed milk in TBST $(20 \mathrm{mM}$ Tris- $\mathrm{HCl}$, $\mathrm{pH}$ 7.5, $150 \mathrm{mM} \mathrm{NaCl}, 0.05 \%$ Tween-20), incubated with the murine monoclonal anti-polyhistidine immunoglobulin $G$ (IgG), rinsed three times with TBST, incubated with the goat anti-mouse IgG conjugated with alkaline phosphatase, rinsed three times with TBST, and detected with the BCIP (5-bromo-4-chloro-3-indolyl phosphate)/NBT (nitro blue tetrazolium) solution.

\section{Synthesis of $\boldsymbol{n}$-hexadecanal}

$n$-Hexadecanal was synthesized following the published procedure [43]. Dess-Martin reagent $(0.96 \mathrm{~g}, 2.3 \mathrm{mmol})$ in one portion was added to the solution of $n$-hexadecanol (0.5 g, $2.1 \mathrm{mmol})$ in $\mathrm{CH}_{2} \mathrm{Cl}_{2}(40 \mathrm{ml})$ in an ice bath. After $n$-hexadecanol was completely gone, the reaction was quenched at $0^{\circ} \mathrm{C}$ by stirring with the saturated $\mathrm{NaHCO}_{3}$ solution (40 mL) containing $\mathrm{Na}_{2} \mathrm{~S}_{2} \mathrm{O}_{3}(3 \mathrm{~g})$ for 10 min to destroy any unreacted Dess-Martin reagent. The reaction mixture was poured into a separatory funnel and extracted with EtOAc ( $3 \times 40 \mathrm{~mL}$ ). The organic layers were pooled and washed with brine $(50 \mathrm{~mL})$, dried over $\mathrm{MgSO}_{4}$ and concentrated. Crude $n$-hexadecanal was purified by silica column chromatography using hexane:ethyl acetate (9:1) as eluent to obtain an oily liquid. The product was confirmed by GC-MS.

\section{Enzyme assay}

All experiments were done at least in duplicate.

\section{(a) For FNR and Fd}

Enzymatic assays for FNR and Fd were done on the Beckman Coulter DU 800 UV/Vis Spectrophotometer at $1 \mathrm{ml}$ scale at $25^{\circ} \mathrm{C}$.

Ferricyanide reductase activity was measured with $\mathrm{NADPH}$ and potassium ferricyanide as the electron donor and acceptor molecules, respectively $[27,44]$. Assays were performed in $50 \mathrm{mM}$ Tris- $\mathrm{HCl}$ ( $\mathrm{pH}$ 8.0) containing $0.7 \mathrm{mM}$ potassium ferricyanide and different $\mathrm{NADPH}$ concentrations $(15-450 \mu \mathrm{M})$ or NADH $(2 \mathrm{mM})$. The reactions were initiated by the addition of $0.02 \mu \mathrm{M}$ FNR. The absorption decrease at $420 \mathrm{~nm}$ (reduction of ferricyanide, $\varepsilon_{420}=1000 \mathrm{M}^{-1} \mathrm{~cm}^{-1}$ ) was recorded to determine steady-state kinetic parameters.

The Fd-mediated cytochrome $c$ reductase activity of FNR was measured with Fd and cytochrome $c$ acting as the intermediate and terminal electron acceptors [30,41]. Assays were carried out in $50 \mathrm{mM}$ Tris- $\mathrm{HCl}$ (pH 7.8) containing $0.01 \mu \mathrm{M}$ FNR and $50 \mu \mathrm{M}$ cytochrome $c$. The reactions were started by the addition of NADPH $(375 \mu \mathrm{M})$. Steady-state kinetic parameters for the Fd-dependent cytochrome $c$ reductase activity were determined by varying the concentrations of Fd $(3-80 \mu \mathrm{M})$ and monitoring the resulting absorption increases at $550 \mathrm{~nm}$ (reduction of cytochrome $c, \varepsilon_{550}=19,100 \mathrm{M}^{-1} \mathrm{~cm}^{-1}$ ).

\section{(b) For ADO 1593}

\section{n-Hexadecanal used as the substrate}

The typical reaction contained $20 \mu \mathrm{M}$ ADO 1593, $80 \mu \mathrm{M}$ ferrous ammonium sulfate, $150 \mu \mathrm{M} n$-hexadecanal in $500 \mu \mathrm{L}$ of $100 \mathrm{mM}$ HEPES (pH 7.2) containing $0.1 \mathrm{M}$ $\mathrm{KCl}$ and $10 \%$ glycerol. $n$-Hexadecanal was made up as the stock solution in $2 \%$ Triton X-100 containing $150 \mu \mathrm{M}$ BSA [16]. The biological reducing system is comprised of either $10.26 \mu \mathrm{M}$ Fd from spinach or PCC7942, 1.8 $\mu \mathrm{M}$ FNR from spinach or PCC7942 and $2 \mathrm{mM}$ NADPH. The chemical reducing system consists of $75 \mu \mathrm{M}$ phenazine methosulfate (PMS) and $750 \mu \mathrm{M}$ NADH. For the biological reducing system, four groups of experiments were set up: (a) $\mathrm{Fd}_{\text {spinach }}$ and $\mathrm{FNR}_{\text {spinach}}$; (b) $\mathrm{Fd}_{\mathrm{PCC} 7942}$ and $\mathrm{FNR}_{\mathrm{PCC} 7942}$; (c) $\mathrm{Fd}_{\text {spinach }}$ and $\mathrm{FNR}_{\mathrm{PCC} 7942}$; (d) $\mathrm{Fd}_{\mathrm{PCC} 7942}$ and $\mathrm{FNR}_{\text {spinach. }}$ The reactions were incubated at room temperature for $1 \mathrm{hr}$ at $200 \mathrm{rpm}$, quenched by the addition of $500 \mu \mathrm{L}$ ethyl acetate and vortexed to extract the hydrocarbon product and the unreacted substrate. One $\mu \mathrm{L}$ of the ethyl acetate layer was injected into GC-MS for analysis. Enzymatic conversion of $n$-hexadecanal into $n$-pentadecane was quantified using the calibration plot of $n$-pentadecane.

An Agilent 5975C GC-MS system equipped with a quadrupole mass detector was used to detect and quantify the hydrocarbons formed in the enzymatic reactions. 
The column employed for hydrocarbon analysis was a HPINNOWax capillary column $(30 \mathrm{~m} \times 0.25 \mathrm{~mm} \times 0.25 \mu \mathrm{m})$. The ethyl acetate extracts of the reaction mixtures were used for GC-MS analysis. The flow rate of the helium carrier gas was $1.0 \mathrm{~mL} / \mathrm{min}$ and the inlet temperature was maintained at $250^{\circ} \mathrm{C}$. Injections were made in the splitless mode and a total flow of $50 \mathrm{~mL} / \mathrm{min}$. The interface temperature was maintained at $250^{\circ} \mathrm{C}$. The oven temperature was held at $40^{\circ} \mathrm{C}$ for $5 \mathrm{~min}$ and then increased to $200^{\circ} \mathrm{C}$ at $25^{\circ} \mathrm{C} / \mathrm{min}$ and finally maintained at $240^{\circ} \mathrm{C}$ for $15 \mathrm{~min}$. Chromatographic data were analyzed using the associated software.

\section{n-heptanal used the substrate}

For determining $k_{\text {cat }}$ value of ADO 1593 against $n$-heptanal, assays were performed in $1.5 \mathrm{~mL}$ gastight vials with a total volume of $500 \mu \mathrm{L}$. The reactions contained $2 \mathrm{mM} \mathrm{n}$ heptanal in $100 \mathrm{mM}$ HEPES buffer (pH 7.2) containing $100 \mathrm{mM} \mathrm{KCl}, 10 \%$ glycerol and $4 \% \mathrm{DMSO}, 5 \mu \mathrm{M}$ ADO, $20 \mu \mathrm{M}$ ferrous ammonium sulphate, $10.26 \mu \mathrm{M} \mathrm{Fd}$ and $1.8 \mu \mathrm{M}$ FNR from PCC7942, $2 \mathrm{mM}$ NADPH (for the cognate reducing system), or $15 \mu \mathrm{M}$ ADO, $60 \mu \mathrm{M}$ ferrous ammonium sulphate, $75 \mu \mathrm{M}$ PMS, $750 \mu \mathrm{M}$ NADH (for the chemical reducing system). The $n$-heptanal stock solution was freshly made in DMSO. After the addition of all the components, the reactions were shaken at $220 \mathrm{rpm}$ at $37^{\circ} \mathrm{C}$. To determine the amount of $n$-hexane produced, a sample of the headspace was collected using a gastight sample lock Hamilton syringe and analysed by GC. The amount of $n$-hexane produced was quantified by the standard curve of known concentrations of $n$-hexane.

GC analysis was performed using an Agilent 7890A instrument equipped with a flame ionization detector and a HP-INNOWax capillary column $(30 \mathrm{~m} \times 0.32 \mathrm{~mm} \times$ $0.25 \mu \mathrm{m})$. The flow rate of the nitrogen carrier gas was $1.1 \mathrm{~mL} / \mathrm{min}$ and the inlet temperature was maintained at $250^{\circ} \mathrm{C}$. Injections were made in the split mode with a split ratio of 2:1 and a total flow of $2 \mathrm{~mL} / \mathrm{min}$. The oven temperature was held at $80^{\circ} \mathrm{C}$ for $2 \mathrm{~min}$ and then increased to $180^{\circ} \mathrm{C}$ at $20^{\circ} \mathrm{C} / \mathrm{min}$, followed by increasing to $210^{\circ} \mathrm{C}$ at $30^{\circ} \mathrm{C} / \mathrm{min}$, and finally maintained at $210^{\circ} \mathrm{C}$ for $2 \mathrm{~min}$. The FID detector was at $300^{\circ} \mathrm{C}$ with a continuous flow of $\mathrm{H}_{2}$ at $30 \mathrm{~mL} / \mathrm{min}$ and air at $350 \mathrm{~mL} / \mathrm{min}$. Chromatographic data were analyzed using the HP Chem station software.

\section{Determination of $\mathrm{H}_{2} \mathrm{O}_{2}$ production during $\mathrm{ADO}$-catalyzed reactions using $n$-hexadecanal used as the substrate}

ADO-catalyzed reactions were set up as above for the cognate reducing system and the surrogate one. After $15 \mathrm{~min}, \mathrm{H}_{2} \mathrm{O}_{2}$ production was determined on Synergy HT Multi-Mode Microplate Reader with the Amplex Red Hydrogen Peroxide/Peroxidase Assay Kit according to the manufacturer's protocol.

\section{Additional files}

\section{Additional file 1: Codon-optimized gene sequence of ADO from} Synechococcus elongatus PCC7942.

Additional file 2: Figure S1. SDS-PAGE analysis of FNR. Figure S2. SDS-PAGE analysis of Fd.

Additional file 3: Figure S3A. Residues involved in FAD binding in FNR from Synechocystis sp. PCC7002 (PDB ID:2B5O). Figure S3B. Sequence alignment of FNRs from PCC6803, PCC7002, and PCC7942.

\section{Abbreviations}

ACP: Acyl carrier protein; ADO: Aldehyde-deformylating oxygenase; Fd: Ferredoxin; FNR: Ferredoxin-NADP ${ }^{+}$reductase; MeOPMS: 1-methyoxy-5methylphenazinium methylsulfate; P450: Cytochrome P450; PMS: Phenazine methosulfate.

\section{Competing interests}

The authors declare that they have no competing interests.

\section{Authors' contributions}

$J Z, X L$, and $J J L$ designed the experiments. JZ performed the experiments, including gene cloning, overexpression, purification, characterization, and enzymatic assays. JZ, XL, and JJL drafted the manuscript. All authors read and approved the final manuscript.

\section{Acknowledgements}

We thank Mr Chenjun Jia, Institute of Biophysics, Chinese Academy of Sciences, for kindly providing data on analytical ultracentrifugation and MALDI-TOF-MS. This work was supported by grants from the National Basic Research Program of China (973: 2011CBA00907) and National Science Foundation of China (31170765).

\section{Author details}

${ }^{1}$ Key Laboratory of Biofuels, Shandong Provincial Key Laboratory of Energy Genetics, Qingdao Institute of Bioenergy and Bioprocess Technology, Chinese Academy of Sciences, No. 189 Songling Road, Qingdao 266101, China.

${ }^{2}$ University of Chinese Academy of Sciences, Beijing 100049, China.

Received: 4 February 2013 Accepted: 3 June 2013

Published: 8 June 2013

\section{References}

1. Daroch M, Geng S, Wang G: Recent advances in liquid biofuel production from algal feedstocks. App/ Energy 2013, 102:1371-1381.

2. Machado IMP, Atsumi S: Cyanobacterial biofuel production. J Biotechnol 2012, 162:50-56

3. Zhang F, Rodriguez S, Keasling JD: Metabolic engineering of microbial pathways for advanced biofuels production. Curr Opin Biotechnol 2011, 22:1-9.

4. Rude MA, Schirmer A: New microbial fuels: a biotech perspective. Curr Opin Microbiol 2009, 12:274-281.

5. Keasling JD, Chou H: Metabolic engineering delivers next-generation biofuels. Nat Biotechnology 2008, 26:298-299.

6. Lee SK, Chou H, Ham TS, Lee TS, Keasling JD: Metabolic engineering of microorganisms for biofuels production: from bugs to synthetic biology to fuels. Curr Opin Biotechnol 2008, 19:556-563.

7. Geddes CC, Nieves IU, Ingram LO: Advances in ethanol production. Curr Opin Biotechnol 2011, 22:312-319.

8. Schirmer A, Rude MA, Li X, Popova E, del Cardayre SB: Microbial biosynthesis of alkanes. Science 2010, 329:559-562.

9. Lu X: A perspective: photosynthetic production of fatty acid-based biofuels in genetically engineered cyanobacteria. Biotechnol Adv 2010, 28:742-746.

10. Tan X, Wang W, Lu X: Production of photosynthetic biofuels by genetically engineering cyanobacteria. Curr Chem Biol 2012, 6:26-31.

11. Rosgaard L, de Porcellinis AJ, Jacobsen JH, Frigaard N-U, Sakuragi Y: Bioengineering of carbon fixation, biofuels, and biochemical in cyanobacteria and plants. J Biotechnol 2012, 162:134-147. 
12. Warui DM, Li N, Nørgaard H, Krebs C, Bollinger JM Jr, Booker SJ: Detection of formate, rather than carbon monoxide, as the stoichiometric co-product in conversion of fatty aldehydes to alkanes by a cyanobacterial aldehyde decarbonylase. J Am Chem Soc 2011, 133:3316-3319.

13. Li N, Nørgaard H, Warui DM, Booker SJ, Krebs C, Bollinger JM Jr: Conversion of Fatty Aldehydes to Alka(e)nes and formate by a cyanobacterial aldehye decarbonylase: cryptic redox by an unusual dimetal oxygenase. J Am Chem Soc 2011, 133:6158-6161.

14. Li N, Chang W, Warui DM, Booker SJ, Krebs C, Bollinger JM Jr: Evidence for only oxygenative cleavage of aldehydes to Alk(a/e)nes and formate by cyanobacterial aldehye decarbonylase. Biochemistry 2012, 51:7908-7916.

15. Das D, Eser BE, Han J, Sciore A, Marsh ENG: Oxygen-independent decarbonylation of aldehydes by cyanobacterial aldehyde decarbonylase: a new reaction of di-iron enzymes. Angew Chem Int Ed 2011, 50:7148-7152.

16. Eser BE, Das D, Han J, Jones PR, Marsh ENG: Oxygen-independent alkane formation by non-heme iron-dependent cyanobacterial aldehye decarbonylase: investigation of kinetics and requirement for an external electron donor. Biochemistry 2012, 50:10743-10750.

17. Krebs C, Bollinger JM Jr, Booke SJ: Cyanobacterial alkane biosynthesis further expands the catalytic repertoire of the ferritin-like 'di-iron-carboxylate' proteins. Curr Opin Chem Biol 2011, 15:1-13.

18. Andre C, Kim SW, Yu X-H, Shanklin J: Fusing catalase to an alkane-producing enzyme maintains enzymatic activity by converting the inhibitory byproduct $\mathrm{H}_{2} \mathrm{O}_{2}$ to the cosubstrate $\mathrm{O}_{2}$. Proc Natl Acad Sci USA 2013, 110:3191-3196.

19. Rogge CE, Fox BG: Desaturation, chain scission, and register-shift of oxygen-substituted fatty acids during reaction with Stearoyl-ACP Desaturase. Biochemistry 2002, 41:10141-10148.

20. Choi YS, Zhang H, Brunzelle JS, Nair SK, Zhao H: In vitro reconstitution and crystal structure of $p$-aminobenzoate $\mathrm{N}$-oxygenase (AurF) involved in aureothin biosynthesis. Proc Natl Acad Sci USA 2008, 105:6858-6863.

21. Barry SM, Kers JA, Johnson EG, Song L, Aston PR, Patel B, Krasnoff SB, Crane BR, Gibson DM, Loria R, Challis GL: Cytochrome P450-catalyzed L-tryptophan nitration in thaxtomin phytotoxin biosynthesis. Nat Chem Biol 2012, 8:814-6.

22. Chun YJ, Shimada T, Sanchez-Ponce R, Martin MV, Lei L, Zhao B, Kelly SL, Waterman MR, Lamb DC, Guengerich FP: Electron transport pathway for a Streptomyces cytochrome P450: cytochrome P450 105D5-catalyzed fatty acid hydroxylation in Streptomyces coelicolor A3(2). J Biol Chem 2007, 282:17486-500.

23. Hannemann F, Bichet A, Ewen KM, Bernhardt R: Cytochrome P450 systems-biological variations of electron transport chains. Biochim Biophys Acta 2007, 1770:330-344.

24. Yang W, Bell SG, Wang H, Zhou W, Hoskins N, Dale A, Bartlam M, Wong L-L, Rao Z: Molecular characterization of a class I P450 electron transfer system from Novosphingobium aromaticivorans DSM12444. J Biol Chem 2012, 285:27372-27384.

25. Bell SG, Dale A, Rees NH, Wong L-L: A cytochrome P450 class I electron transfer system from Novosphingobium aromaticivorans. Appl Microbiol Biotechnol 2010, 86:163-175.

26. Sasaki M, Akahira A, Oshiman K, Tsuchido T, Matsumura Y: Purification of cytochrome P450 and ferredoxin, involved in bisphenol a degradation, from Sphingomonas sp. Strain AO1. Appl Environ Microbiol 2005, 71:8024-8030.

27. Korn A, Ajlani G, Lagoutte B, Gall A, Sétif P: Ferredoxin:NADP ${ }^{+}$oxidoreductase association with phycocyanin modulates its properties. J Biol Chem 2009, 284:31789-31797.

28. Thomas JC, Ughy B, Lagoutte B, Ajlani G: A second isoform of the ferredoxin:NADP oxidoreductase generated by an in-frame initiation of translation. Proc Natl Acad Sci USA 2006, 103:18368-73.

29. van der Plas J, de Groot RP, Woortman MR, Cremers F, Borrias M, van Arkel GA, Weisbeek PJ: Genes encoding ferredoxins from Anabaena sp. PCC 7937 and Synechococcus sp. PCC7942: structure and regulation. Photosyn Res 1988, 18:179-204

30. Poncelet M, Cassier-Chauvat C, Leschelle X, Bottin H, Chauvat F: Targeted deletion and mutational analysis of the essential (2Fe-2S) plant-like ferredoxin in Synechocystis PCC6803 by plasmid shuffling. Mol Microbiol 1998, 28:813-821.

31. Catalano-Dupuy DL, Musumeci MA, López-Rivero A, Ceccarelli EA: A highly stable plastidic-type Ferredoxin-NADP $(H)$ reductase in the pathogenic bacterium leptospira interrogans. PLoS One 2011, 6:e26736.
32. Zanetti $G$, Arosio P: Solubilization from spinach thylakoids of a higher molecular weight form of ferredoxin-NADP ${ }^{+}$reductase. FEBS Lett 1980 111:373-376.

33. Medina M, Martínez-Júlvez M, Hurley JK, Tollin G, Gómez-Moreno C: Involvement of glutamic acid 301 in the catalytic mechanism of Ferredoxin-NADP ${ }^{+}$reductase from anabaena PCC 7119. Biochemistry 1998, 37:2715-2728

34. Tejero J, Martínez-Júlvez M, Mayoral T, Luquita A, Sanz-Aparicio J, Hermoso JA Hurley JK, Tollin G, Gómez-Moreno C, Medina M: Involvement of the pyrophosphate and the 2'-Phosphate binding regions of Ferredoxin-NADP ${ }^{+}$ reductase in coenzyme specificity. J Biol Chem 2003, 278:49203-49214.

35. Floß B, Igloi GL, Cassier-Chauvat C, Mühlenhoff U: Molecular characterization and overexpression of the petF gene from Synechococcus elongatus: evidence for a second site of electrostatic interaction between ferredoxin and the PS I-D subunit. Photosynth Res 1997, 54:63-71.

36. Piubelli L, Aliverti A, Bellintani F, Zanetti G: Spinach ferredoxin I: overproduction in Escherichia coli and purification. Protein Expr Purif 1995, 6:298-304.

37. Baumann B, Sticht $H$, Schärpf $M$, Sutter $M$, Haehnel W, Rösch P: Structure of Synechococcus elongatus $\left[\mathrm{Fe}_{2} \mathrm{~S}_{2}\right.$ ] Ferredoxin in Solution. Biochemistry 1996, 35:12831-12841.

38. Ewen KM, Kleser M, Bernhardt R: Adrenodoxin: the archetype of vertebrate-type [2Fe-2S] cluster ferredoxins. Biochim Biophys Acta 1814, 2011:111-125

39. Medina M, Gómez-Moreno C: Interaction of ferredoxin-NADP + reductase with its substrates: optimal interaction for efficient electron transfer. Photosynth Res 2004, 79:113-131.

40. Bell SG, McMillan JHC, Yorke JA, Kavanagh E, Johnson EOD, Wong L-L: Tailoring an alien ferredoxin to support native-like P450 monooxygenase activity. Chem Commun 2012, 48:11692-11694.

41. O'Keefe DP, Gibson KJ, Emptage MH, Lenstra R, Romesser JA, Litle PJ, Omer CA: Ferredoxins from Two Sulfonylurea Herbicide Monooxygenase Systems in Streptomyces griseolus. Biochemistry 1991, 30:447-455.

42. Sambrook J, Fitsch EF, Maniatis T: Molecular Cloning: A Laboratory Manual. Cold Spring Harbor Press: Cold Spring Harbor; 1989

43. de Lera AR, Okamura WH: 19,19,19- and 20,20,20-trimethylretinal: Side chain tert-butyl substituted retinals. Tetrahedron Lett 1987, 28:2921-2924.

44. Zanetti G, Curti B: Ferredoxin-NADP ${ }^{+}$Oxidoreductase. Methods Enzymol $1980,69: 250-255$

\section{doi:10.1186/1754-6834-6-86}

Cite this article as: Zhang et al:: Conversion of fatty aldehydes into alk (a/e)nes by in vitro reconstituted cyanobacterial aldehyde-deformylating oxygenase with the cognate electron transfer system. Biotechnology for Biofuels 2013 6:86.

\section{Submit your next manuscript to BioMed Central and take full advantage of:}

- Convenient online submission

- Thorough peer review

- No space constraints or color figure charges

- Immediate publication on acceptance

- Inclusion in PubMed, CAS, Scopus and Google Scholar

- Research which is freely available for redistribution 